\title{
Interactive Voice Response Kiosk
}

\author{
Natasha Brahme ${ }^{1}$, Ayushi Malhotra ${ }^{2}$, Harsh Panchal ${ }^{3}$, Chirag Waghela ${ }^{4}$, Dr. V.C. Kotak ${ }^{5}$ \\ Student, IT, Shah \&Anchor Kutchhi Engineering College, Mumbai, India 1, 2, 3, 4 \\ Vice Principal, Shah \&Anchor Kutchhi Engineering College, Mumbai, India ${ }^{5}$
}

\begin{abstract}
Interactive Voice Response Kiosk is built using artificial algorithms that analyses user's queries and understand user's message. In our case, we're using the bot for college purposes - to answer the queries of the student based on web system. Students or any visitor or faculty just has to query through the bot which is used for chatting. Students can chat using any format, there is no specific format the user has to follow. The System uses built in artificial intelligence to answer the query. The answers are appropriate to what the user queries. The User can query any college related activities through the system - be it academic, extra-curricular activities, sports, results, certified courses etc. The user does not have to personally go to the college for enquiry. The System analyses the question and then answers to the user. The system answers to the query as if it is answered by the person. With the help of artificial intelligence, the system answers the query asked by the students. The system replies using an effective Graphical user interface. The student \& faculty just has to register himself to the system and has to login to the system. After login, they can have access to the various helping pages the bot through which the students can chat by asking queries related to college activities. The project also provides another two tabs called Visitor \& Parent. The visitor just has to go the visitor tab and get the visitor slip printed. The visitor has to fill all the details asked \& then save it. Unless it's saved, the slip cannot be printed. The functionality of the parent is the same as that of the student. The only difference is that the parent does not login. It just asked the queries. But for that, the parent has to go to the respective tab. If the parent wants to meet someone, then it becomes the visitor. So the parent will have to print the receipt after asking the required question. The student can query about the college related activities online with the help of this web app.
\end{abstract}

Keywords: HTML, CSS, PHP, MySQL.

\section{INTRODUCTION}

Traditional web-bots exist; web-bots were created as text The paper further is divided into the following sections: based web-friends, an entertainer for a user. Furthermore, 2. Our Approach, and separately there already exists enhanced rich site 3. System Implementation, summary (RSS) feeds and expert content processing 4. System Architecture, systems that are accessible to web users. Text-based web- 5. Results, bots can be linked to function beyond an entertainer as an 6. Conclusion $\&$ informer, if linked with, amongst others, RSS feeds and or 7. References expert systems. Such a friendly bot could, hence, also function as a trainer providing realistic and up-to-date responses.

The convenience could be improved if the system is not only text based but also voice-based \& voice trained. To create a more user accessible chat system; a simpler input method using text is introduced; creating and catering for a more personal and convenient experience. The process of an online chat system would follow a client server approach which acquires the signal and streams it to a server. The input text is then processed and a response is generated. This process places a large processing requirement on the server's processor and memory resources. This limitation is even more evident when a large number of users are to $b$ simultaneously accommodated on the system. Interactive Voice Response Kiosk is a web based system designed to accept a user's query and respond to those queries, maximizing its chances of correct responses at some goal. This problem is addressed in this paper.

\section{OUR APPROACH}

IVRK is based on chatbots. Different types of chatter bots already implemented are A.L.I.C.E, ELIZA, and Captain KIRK. IVRK will consist of a user friendly interface providing three logins respectively:

1. Student Login

2. Admin Login

3. Faculty login

The system mainly concentrates on user input. As the system gets the input, it will process the input depending on the different query keywords which will be compared with the keywords stored in the database. It mainly aims at providing almost accurate response to the query. If the users query is not satisfied by the system the user can mark the answer as invalid. Once the administrator logs in to the system the administrator will be able to view the invalid marked questions. The administrator can then 
decide whether to consider the marked answer as invalid or not and then can choose to update the database or delete the invalid marked questions. The administrator can enter questions on basis of keywords and enter answers in the database.

The other three tabs provided are the "Parent", the "Visitor" \& the "Faculty" tab. The visitor does not has to login. $\mathrm{He} / \mathrm{she}$ just has to fill the details mentioned on the visitor tab, print the receipt and he is good to go. The parent can directly ask about anything without logging \& printing. The functionality of parent is similar to the student functionality. The difference is that the parent tab does not have to login. Another tab is the "Faculty" tab. The functionality of this is same as that of the student. Only the personal details of the faculty like the contact number is hidden from the student \& the visitor but it's visible to the other faculties at the same time.

\section{DATABASE}

A relational database management system (RDBMS) is a program set that is employed to define, manage, and process a database. The database is a structure that is built to be functioned as data storage. MySQL is a server database or RDBMS software that can manage the database and can store data in many numbers. It can be accessed by multi-user and can do multi-threaded. The chatbot consists of core and interface accessing that core. The core is in RDBMS being database. The database consists of tables to store knowledge. This project consists of a database that maintains the logs of the qts asked, the invalid marked qts, the questions and the desired answers to it. The data is stored in a tabular format containing columns namely main keyword, k1 ,k2 ,k3,k4.

\section{TABLES}

The table blocks consist of main tables as scheme and supporting tables i.e. main keyword, k1, k2, k3, k4. Also there is a flag content that specifies whether that particular question should be visible to the student or not. The main keyword is the important keyword which is surely present in the question asked and the other columns contain the subordinate keywords that makes it easier for the bot to reply during the text matching process. The keyword stores list of keywords that is probably found in the user input. The keywords are used to narrow the range of pattern searching. This project consists of qts asked which stores the history of qts asked, invalid qts which shows questions that are marked by the users which is visible only to the admin.

\section{SYSTEM ARCHITECTURE}

\section{Client}

The client in this project is the webpage where the user can either $\log$ in depending on the kind of the user i.e. faculty or the student or just ask the question directly on the site to which the bot will reply with an answer in a oice and text format. The webpage i.e the front end is $\}$ developed using basic html \&v html 5.0 tags, bootstrap, css and c\# on Microsoft visual studio whereas the backend used is MySQL as a database where the history of the answers provided, the questions ask4ed \& the invalid marked questions are stored. The users interact with the chat bot with the help of this webpage.

\section{SERVER}

The server is the local host server. Server response generation can be broken down into two categories: data retrieval and information output. The core focus of this paper is to improve the information output by generating a response that is relevant to the request, factual and personal. This requires aspects of news and an intelligent algorithm to generate informative and user specific responses. The server side includes the Chatbot module and the Text-to-Speech module, including the Natural Language Processing and the Digital Signal Processing modules. On the client side the user interacts with the Talking Head introducing his questions in a written chat form.

\section{TEXT-TO-SPEECH (TTS)}

Speech synthesis is the artificial production of human speech. A computer system used for this purpose is called a speech computer or speech synthesizer, and can be implemented in software or hardware products. A text-tospeech (TTS) system converts normal language text into speech; The Text To Speech (TTS) process is obtained by means of a voice synthesizer, which is a system that produces the vocal signal using the phonetic transcription of the analysed sentence. The aim is to produce a speech which should seem the most natural through two modules. The first one is the Natural Language Processing (NLP) module, capable of producing a phonetic transcription of the text using information about the prosody. The second one is the DSP (Digital Signal Processing) module that translate the symbolic information obtained from the NLP module.

if (Session["Bot"] == "Yes")

$$
\{
$$

SpVoice voice;

voice $=$ new $\operatorname{SpVoice}() ;$ voice. Volume $=100 ; / / 0 \ldots 100$

voice. Rate $=-2 ; \quad / / \quad-10 \ldots 10 \quad$ TextBox8.Text = Session["Ques"].ToString(); TextBox13.Text = Session["Ans"]

.ToString().Replace("'", "');

if $($ TextBox13.Text $=="$ ")

TextBox13.Text $=$ "Sorry we do not have answer for that question"; 


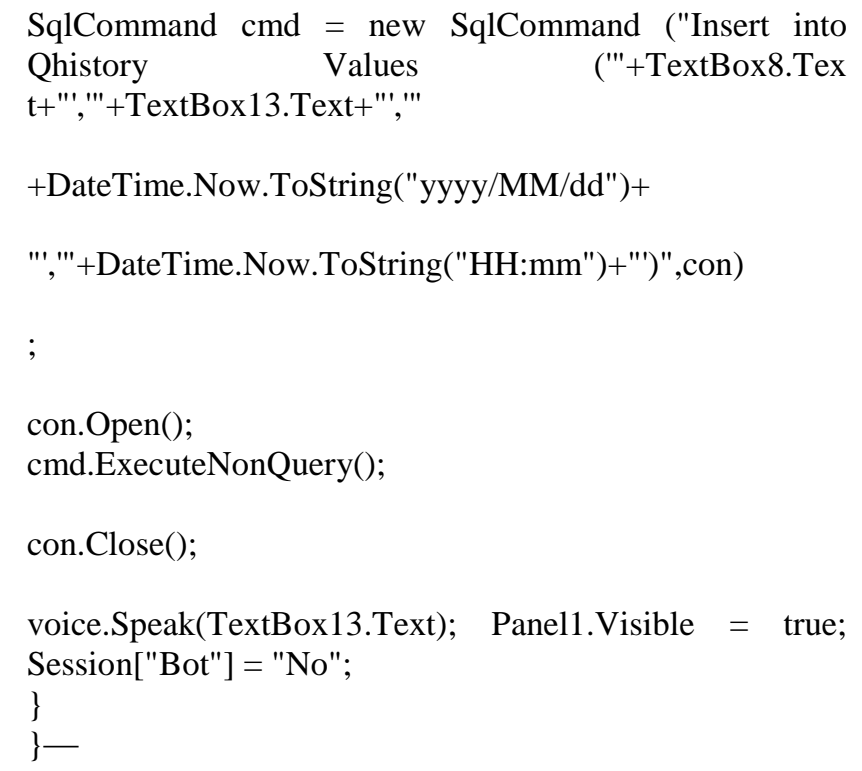

\section{RESULTS}

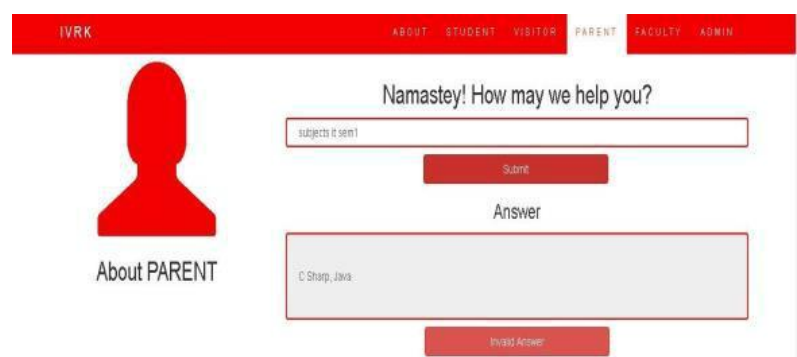

Fig 1

The above image shows an example of the proposed project. The user, in this case is the parent who enters the desired question it wants to ask the bot. The bot replies in a textual and vocal format. Similarly, the student or the faculty can login respectively.

\section{CONCLUSION}

The development of chat bot applications in various programming languages had been done with making a user interface to send input and receive response. This project helps in giving the response in a text as well as a voice format for better communication with the help of keywords matching technique. Chat bots are in use in various different fields. Recently, a company came up with an idea in the retail industry such that to every display piece, the bot will be associated. The bot will be replying to all the queries the customer has about that particular piece like the quality, material, its make, the size etc. This idea is still in thoughts and not yet implemented.

\section{REFERENCES}

[1] Stork D., Henneke M. (Eds.) (1996), Speech-Reading by Humans and Machine: Models, Systems and Applications, SpringerVerlag,New York
[2] [Colby, 1973] K. M. Colby. Simulation of Belief systems. In Schank and Colby (Eds.) Computer Models of Thought and Language, pp.251-286, 1973.

[3] C. Erdogan, H. Nusret Bulus, and B. Diri, "Analyzing The Performance Differences Between Pattern Matching and Compressed Pattern Matching on Texts," Proc. IEEE of 2013 International Conference on Electronics, Computer and Computation (ICECCO), 2013, pp. 135-138, doi:10.1109/ICECCO.2013.6718247.

[4] J. P. McIntire, L. K. McIntire, and P. R. Havig, "Methods for Chatbot Detection in Distributed Text-Based Communications," Proc. IEEE of 2010 International Symposium on Collaborative Technologies and Systems (CTS), 2010, pp. 463-472, doi:10.1109/CTS.2010.5478478.

[5] J. P. McIntire, L. K. McIntire, and P. R. Havig, "Methods for Chatbot Detection in Distributed Text-Based Communications," Proc. IEEE of 2010 International Symposium on Collaborative Technologies and Systems (CTS), 2010, pp. 463-472, doi:10.1109/CTS.2010.5478478. 